\title{
A Comparison of Artificial Driving Sounds for Automated Vehicles
}

\author{
David Beattie \\ ITT Research Group, SEBE \\ Glasgow Caledonian University \\ Glasgow, G4 0BA,UK \\ david.beattie@gcu.ac.uk
}

\author{
Lynne Baillie \\ Department of Computing \\ Heriot-Watt University \\ Edinburgh, EH14 4AS, UK \\ lynne.baillie@hw.ac.uk
}

\author{
Martin Halvey \\ Department of CIS \\ University of Strathclyde \\ Glasgow, G1 1XQ, UK \\ martin.halvey@strath.ac.uk
}

\begin{abstract}
As automated vehicles currently do not provide sufficient feedback relating to the primary driving task, drivers have no assurance that an automated vehicle has understood and can cope with upcoming traffic situations [16]. To address this we conducted two user evaluations to investigate auditory displays in automated vehicles using different types of sound cues related to the primary driving sounds: acceleration, deceleration/braking, gear changing and indicating. Our first study compared earcons, speech and auditory icons with existing vehicle sounds. Our findings suggested that earcons were an effective alternative to existing vehicle sounds for presenting information related to the primary driving task. Based on these findings a second study was conducted to further investigate earcons modulated by different sonic parameters to present primary driving sounds. We discovered that earcons containing naturally mapped sonic parameters such as pitch and timbre were as effective as existing sounds in a simulated automated vehicle.
\end{abstract}

\section{Author Keywords}

Automated Vehicles; Auditory Displays; Driving Simulator; Earcons; Auditory Icons; Speech

\section{ACM Classification Keywords}

H5.2 [Information interfaces and presentation]: User Interfaces. -Auditory (non-speech) feedback, evaluation/methodology

\section{INTRODUCTION}

The benefits of vehicular automation include improvements in road safety, traffic efficiency and enriched journey experience. They also contribute to societal gains such as improved energy efficiency via more precise vehicular control [11]. In order to obtain these benefits, a number of important challenges must be addressed [24]. These challenges exist not only to automated vehicles on a collective level, where issues such as traffic management, road safety and legal concerns arise.

\footnotetext{
Permission to make digital or hard copies of all or part of this work for personal or classroom use is granted without fee provided that copies are not made or distributed for profit or commercial advantage and that copies bear this notice and the full citation on the first page. Copyrights for components of this work owned by others than ACM must be honored. Abstracting with credit is permitted. To copy otherwise, or republish, to post on servers or to redistribute to lists, requires prior specific permission and/or a fee. Request permissions from Permissions@acm.org. UbiComp '15, September 07-11, 2015, Osaka, Japan (C) 2015 ACM. ISBN 978-1-4503-3574-4/15/09 $\square \$ 15.00$ DOI: http://dx.doi.org/10.1145/2750858.2807519
}

There are also challenges concerning the control and operation of automated vehicles on an individual level and what impact this has on driver/vehicle interactions.

Traditional manually controlled vehicles require a driver to continuously operate primary driving tasks, resulting in real-time feedback that connects driver and vehicle. This connection ensures drivers are aware of any necessary actions that must be performed during challenging driving situations [39]. In contrast, automated vehicles provide no connection to primary driving tasks. Drivers are placed in a supervisory role where they are required to monitor the system performing the task of driving [38]. While this reduces the mental workload for drivers [5], their situational awareness is also greatly reduced [39]. This is because no feedback is presented to reassure drivers that an automated vehicle has understood and can cope with upcoming traffic situations [16]. Coupled with an unnatural driving style, this lack of feedback is a direct result of the control shift introduced by automated vehicles [16]. Conversely, drivers are not burdened with the high visual demands of driving when travelling in an automated vehicle, enabling them to freely carry out non-driving tasks [21]. This freedom combined with the need to present feedback relating to the primary driving task will result in new interaction methods not currently present in manually controlled vehicles [24].

Combined with the move towards fully automated vehicles, modern vehicle manufacturers strive to create quieter cars, both internally and externally. With the emergence of hybrid and electric vehicles, familiar mechanical sounds to which drivers are accustomed are abandoned [34]. Research has already been conducted regarding the sound of vehicles using alternative propulsion technologies [27,28,33,34]. However, diminishing auditory feedback has been shown to cause unexpected behaviours such as faster driving and reduced situational awareness [39]. In order to re-establish the connection between driver and vehicle, we believe that automated vehicles should provide a level of feedback equivalent to that of their manual counterparts. This feedback should convey the intended actions of an automated vehicle whilst it undertakes the task of driving. Auditory feedback may be useful for this as previous work has shown that novice drivers tend to utilise visual aids sub optimally during stressful situations [22]. Implicit auditory feedback may create a more satisfactory driving experience and re-establish a sense of control in automated vehicles. 
For these reasons we chose to investigate how to design an auditory display that may be used to diminish the control shift introduced by automated vehicles. This paper presents findings from 2 driving simulator studies. The first study compared earcons, auditory icons and speech with existing vehicle sounds during manual and automated driving conditions. Each sound cue type was comprised of the following low-level driving sounds: acceleration, braking/deceleration, gear-changing and indication [4]. A second study based on the findings from our initial study was then conducted, investigating the sonic parameters: timbre, pitch, repetition and volume. The primary aim of these studies was to determine whether alternative sound cue types are more beneficial than existing vehicle sounds when presenting primary driving information in automated vehicles. We explore the potential benefits brought about through the use of alternative sound cue types for information presentation. We also highlight which sonic parameters are most appropriate when designing artificial driving sounds.

\section{RELATED WORK}

\section{Automated Vehicles}

The automation of primary driving tasks is fast becoming a reality due to an ever-increasing trend in in-vehicle computing [38]. With backing from multiple car manufacturers and events such as the DARPA Urban Challenge [32], in certain parts of the world automated vehicles are already on our roads. The development in the computerised control and operation of automated vehicles leads to a number of issues that must be addressed to ensure automated vehicles are accepted by drivers. 5 levels of vehicle automation have been defined by the National Highway Traffic Safety Administration (NHTSA) of the USA [24]. Level 0 is defined as the human driver having total control of the vehicle's primary controls at all times. In contrast, level 4 sees the vehicle perform all safety critical driving functions, requiring only destination input by the driver. The driver would not be expected to be available for control at any point during their journey. Levels 1, 2 and 3 pertain to increasing levels of automation from single function automation (1) to limited self-driving automation (3). It is important to point out that currently, automated vehicles adhere to level 3 automation and require the driver for occasional control provided sufficient transition time is given. This work focuses on levels 0 (No Automation) and 4 (Full Self-Driving Automation). These extremes were selected as we hoped they would give us clean and clear results. Modern luxury cars now have a mixture of automation levels 2 and 3 and many prototype level 3 vehicles now exist. By comparing levels 0 and 4, we believe our findings can inform the design of auditory displays that feature in automation levels 2 and 3 as they are on the continuum from level 0 to 4 .

\section{In-Vehicle Auditory Feedback}

Advanced Driver Assistance Systems (ADAS) are a step in the journey towards fully automated vehicles. ADAS can enhance driver perception, measure driver attention, act as an automated safety control and provide alerts to potentially hazardous driving situations [13]. In the case of providing alerts to drivers, ADAS often utilise auditory feedback as a mode of information presentation. Presenting information via the auditory modality is beneficial for ADAS as it is omnidirectional and not subject to specific head or body movement [31]. In the context of safety critical updates, the auditory modality can be an effective alternative to the visual modality particularly as it has been shown drivers do not always pay attention to visual aids whilst undertaking the primary driving task [22].

In ADAS, warning signals to safety critical information is most often presented in the form of auditory feedback $[10,29,30]$. Campbell et al. state that warning signals in ADAS can be produced using: simple tones, earcons, auditory icons and speech [9]. In particular, there is a wealth of research related to simple auditory alerts using tones at varying frequencies. A study by Lin et al. [18] found that higher tones of $1750 \mathrm{~Hz}$ and $3000 \mathrm{~Hz}$ produced faster driver response times in comparison to $500 \mathrm{~Hz}$. Earcons, for example Windows Explorer program events, are short audio messages consisting of structured musical tones [6], and have been shown to be particularly effective when information must be communicated via the auditory modality [7]. In comparison, auditory icons, such as the Apple 'trash' sound, are natural pre-recorded sounds that relate to a specific event, object or action which are then mapped to system messages and objects [23]. It has been shown that auditory icons are an effective solution when designing sounds in order to convey perceived meaning and control a driver's actions [20]. In the context of making a phone call, Janssen et al. [15] used auditory icons such as real driving sounds and heart beats as direct and indirect cues to convey a drivers busyness to a caller. Their study showed the provision of auditory cues to be more effective than when no audio cues were presented. Another benefit of auditory icons is that they require little to no training period as they provide an easy to comprehend connection between sound and system event [17]. In contrast, abstract sounds such as earcons often require a learning period as their perceived meaning may not be understood by all listeners [35]. However, earcons are advantageous when abstract events must be conveyed by auditory feedback, as they are not required to relate directly to a specific event, object or action unlike auditory icons. While speech based auditory displays in vehicles cause a reduction in visual attention to the "road ahead", there has been considerable research on their use within ADAS [2,36,37]. It is necessary to point out that a fully automated vehicle requires no human operation, negating the requirement for visual attention to the "road ahead" [14]. Because of this, there is a need to determine whether speech based auditory feedback is still perceived as a distraction. For this reason we have chosen to include speech in our study. 


\section{Sound Design for Electric Vehicles}

Vehicles propelled by traditional internal combustion engines (ICEs) produce familiar and instantly recognisable sounds [34]. As such, drivers and pedestrians are almost automatically aware of the vehicles location, direction and its current state, for example accelerating or braking [40]. However, with advances in alternative propulsion technologies such as hybrid and electric, the once familiar sound of an ICE is no longer present. The introduction of electric vehicles (EVs) impacts both pedestrian and driver. Pedestrian safety concerns arise as they may not hear an EV approaching. Drivers may also no longer be aware that an $\mathrm{EV}$ is running due to the lack of familiar auditory feedback [28]. Research by Wogalter et al. [40] showed that nearly $80 \%$ of 318 participants preferred an EV to produce a traditional ICE sound or an artificial "hum" sound. This suggests that while drivers requested familiar driving sounds, they may also be open to hearing artificial driving sounds if they mimic an ICE. As EV's become more prevalent, it is important to consider that future automated vehicles will use these alternative propulsion technologies. It is also necessary to determine whether automated vehicle should present auditory feedback related to the driving task and how this feedback should be presented.

\section{STUDY 1}

The purpose of the first study was to determine what effect the presentation of alternative primary driving sounds has on drivers of automated vehicles. We wanted to investigate how to enhance awareness to the intended actions of an automated vehicle. We achieved this by conducting a user study that aimed to answer three research questions:

S1.Q1: Do alternative sound cue types provide benefits over existing vehicle sounds for information presentation?

S1.Q2: Must drivers continue hearing existing vehicle sounds to ensure a sense of control is maintained in automated vehicles?

S1.Q3: Which sound cue type is most preferred by participants?

\section{Driving Task and Sound Cue Types}

Study 1 featured 2 driving conditions (manual and automated) and 4 different sound cue types (existing vehicle sounds, earcons, auditory icons, speech). Thus, participants undertook 8 driving scenarios lasting 2 minutes each, following the procedure of Beattie et al. [5], to ensure the associated task load per scenario was experienced without causing fatigue. During the manual condition (NHTSA Level 0 [24]), participants controlled an Open Driving Simulator (OpenDS) via the steering wheel and pedals. Participants were presented with an identical scenario, 'Paris', which comes bundled with OpenDS. This scenario features a day-time virtual environment consisting of inter-connected single-lane streets containing various traffic obstacles during typical good weather conditions. OpenDS was selected as it provided a good level of immersion whilst also being easily configurable for our different sound cue types. A number of automated vehicles also navigated the environment on pre-defined routes. Due to the inclusion of these vehicles, participants would often be required to react to sudden events such as crashes and other vehicles making turns. Depending on the chosen route through the environment, their interaction with such events would vary. Whilst driving, participants were presented with a first-person view in order to provide a real-world driving position and were required to drive on the right hand side of the road, as this was the default setup.

In order to produce a simulated automated vehicle journey (NHTSA Level 4 [24]), we were guided by the work of Nyeste and Wogalter [40] where videos were shown of a Toyota Prius driving whilst different sound types were played back to users. Our video clips lasted 2 minutes and featured a series of driving manoeuvres again within the 'Paris' scenario. During the automated stage of our experiment participants were asked to sit and observe the journey, paying attention to the sounds, until the scenario completed. The 4 sound cue types each contained the 4 major primary driving sounds: acceleration, braking, indication and gear changing. All created sounds are available from: http://www.ittgroup.org/file-repository.

\section{Existing Vehicle Sounds}

The first sound cue type Existing Vehicle Sounds featured a group of sounds captured from a previous study by Beattie et al. [5]. These sounds were pre-recorded mechanical vehicle sounds from a Volkswagen Polo and elicited the typical sonic characteristics participants would commonly hear whilst controlling their own vehicle. Acceleration and deceleration/braking sounds altered in frequency as the participant depressed/released the accelerator pedal mimicking a real vehicle under similar conditions. The gear change sound would trigger as participants clicked the paddles on the Logitech steering wheel. The indicator sound was triggered via another button on the steering wheel and would repeat until the button was pressed again.

\section{Earcons}

The earcons for the study followed the design guidelines laid out by Brewster et al. [7]. Each were created to replicate the qualities of existing vehicle sounds. For instance, the acceleration/deceleration sound was a continuous synthesised sine-wave tone akin to that of the Existing Vehicle Sounds acceleration/deceleration. The sound contained multiple harmonics with a fundamental frequency of $700 \mathrm{~Hz}$ that was linked to the vehicle's idling RPM. As the RPM of the vehicle increased so too did the frequency of the sound. This was also the case for the rhythm of the tone as the frequency of oscillation increased or decreased with RPM. The inclusion of these effects produced a sound that modulated in a familiar fashion to what participants would already be used to and sounded somewhat similar to an ICE vehicle. The indicator sound mimicked the repetition of the Existing Vehicle Sounds. The synthesised sine-wave tone contained multiple harmonics with a fundamental frequency of $1500 \mathrm{~Hz}$. The sound did 
not change in pitch and repeated until turned off by the driver. 2 earcons for gear changing featured a synthesised saxophone sound. The "gear up" sound featured two ascending notes in $\mathrm{C}$ Major scale beginning on note $\mathrm{C} 5$ $(554 \mathrm{~Hz})$. The "gear down" sound featured two descending notes in $\mathrm{C}$ Minor scale again beginning on note $\mathrm{C} 5$. Each sound played once per gear change.

\section{Auditory Icons}

In contrast to our earcons, each auditory icon was a short non-continuous pre-recorded sound that conveyed a particular action as suggested by Mynatt [23]. The acceleration sound featured revving of an engine that lasted 3 seconds. A tyre screech was used for the braking sound again lasting 3 seconds. The accelerator and brake sounds were triggered by the vehicle's speed state changes and looped every 2 seconds to provide repeated updates of the vehicles current state. 2 seconds was chosen to ensure that when looped, our sounds would not cause any annoyance that has been shown with shorter looping times [1]. The indicator sound was a sped up version of the indicator sound used for the Existing Vehicle Sounds cue type. The sound was triggered once on the indicator being activated but did not repeat. Finally, a 3 second clip of a vehicle changing gears comprised the gear changing auditory icon.

\section{Speech}

The phrases for the speech cue type were captured from an online text-to-speech software ${ }^{1}$ with a female voice stating each particular action. For example, whilst the vehicle was accelerating, the word "accelerating" was heard. Both the acceleration and braking sounds repeated every 2 seconds similar to the Auditory Icons. Gear changing featured the voice stating the current action. For instance if the participant/vehicle changed up a gear, the voice would state "gear up". This was repeated for the indicator sound where the voice would state, "turning < direction>". All speech messages were kept as short as possible, as advised by Vollrath [37].

\section{Equipment}

The study took place in a quiet University room, where participants sat on a padded chair in front of a desk with a 32-inch TV screen (Toshiba 32KVB). A laptop computer running the OpenDS software was connected via HDMI cable. OpenDS is primarily designed with the intention to be used for research purposes and is built on the JMonkeyEngine $^{2}$ [19]. OpenDS facilitates a desktop driving environment when combined with steering wheel and pedal controls to provide an immersive environment from which to conduct analysis. It does however lack inertial feedback that is present during real-world driving as no haptic feedback is provided by OpenDS by default.

\footnotetext{
${ }^{1}$ http://www.naturalreaders.com

${ }^{2}$ http://www.jmonkeyengine.org
}

Sound was delivered monaurally through a set of headphones (Grado SR80i). Participants controlled the vehicle during the manual scenarios via a Logitech DrivingForce $\mathrm{GT}^{3}$ gaming steering wheel. Steering wheel buttons were mapped to the indicator, gear up/gear down and ignition on/off functions.

\section{Participants}

14 participants (10 male, 4 female) aged 18 to 56 $($ mean=32) took part in the study, all with valid driving licenses. 9 of 14 participants were from outside the UK and stated they had previous experience driving on both sides of the road. Their mean driving frequency range was 1 to 3 hours per week. 11 participants owned or had regular use of a vehicle. 2 participants were experienced with automatic transmissions and the rest with manual transmissions. Participants were recruited via email and word of mouth.

\section{Procedure}

Participants were first given an introduction to the study then asked if they wished to continue before completing a short pre-evaluation questionnaire. Participants were then presented with the driving simulator software and briefed on the controls. They were given some time to become accustomed to driving within the simulator before the main phase of the study commenced. Driving conditions were counterbalanced with a randomised order of sound cue types presented to each participant. This ensured any possible order effects were minimised. Each participant undertook 8 scenarios in total lasting 2 minutes each. During the scenarios, participants were told to pay specific attention to the sounds within each scenario. To ensure this was upheld, direct observations were taken by the lead researcher during the timing of each scenario. Between each scenario participants were given approximately 5 minutes to complete each of the subjective questionnaires and Baillie's emotion wheel [3]. Upon completion of the scenarios participants were then provided with a short postevaluation questionnaire. The total length of each session was approximately 1 hour and 15 minutes.

\section{Data Gathering Methods}

We employed 4 separate data gathering methods, which were completed after each of the 8 scenarios. First, a Driving Activity Load Index (DALI) questionnaire [25] was presented. The DALI is a subjective workload measurement tool that is based on the NASA-TLX but tailored towards the driving context. Its use enabled us to ensure participant workload was measured during each driving condition and sound cue type. Next, a system usability scale (SUS) was presented to participants that had been adapted so participants could evaluate the usability of the sounds only as opposed to the driving simulator itself.

3 http://gaming.logitech.com/en-gb/product/driving-forcegt-gaming-wheel 
The SUS is a 10-item questionnaire with 5 possible responses ranging from; Strongly Disagree to Strongly Agree. Six 5-point Likert scale questions were given to obtain numerical qualitative data relating specifically to both studies objectives. The questions were as follows:

1. Did the sound cue type presented in this scenario enhance your awareness of the vehicle's actions?

2. Did the sound cue type presented in this scenario enhance your awareness of your own actions?

3. Did you feel in total control of the vehicle throughout this scenario?

4. Did you feel the vehicle provided you with the necessary sound cues to ensure that you were aware of its intended actions?

5. Did you find the sound cue type presented in this scenario to be a distraction to the primary driving task?

6. How satisfied are you with the sounds of the vehicle in this scenario?

Finally, to obtain an insight into participant emotional responses Baillie et al.'s emotion wheel was presented [3].

\section{Study 1 Results}

DALI and SUS results were analysed using 2-way repeated measure ANOVAs, with driving scenario (2 levels) and sound cue type (4 levels) as factors. Likert scale-rated questions were analysed using Friedman's analysis of variance and Wilcoxon signed-rank tests, as the data was not normally distributed. Pair-wise comparisons for sound cue types were conducted with a Bonferroni correction applied, resulting in a significance level set at $\mathrm{p}=0.0083$.

\section{DALI Workload Analysis}

A significant effect on workload was found $\left(\mathrm{F}_{3,39}=12.316\right.$, $\mathrm{p}<0.001)$. Pairwise comparisons showed that Existing Primary Driving Sounds $(\mathrm{p}=0.001)$ and Earcons $(\mathrm{p}=0.004)$ produced significantly lower workload scores in comparison to Auditory Icons. No other differences were found when comparing sound cue types. This shows that Speech and Earcons did not cause a workload increase in comparison to Existing Vehicle Sounds. This suggests that the utilisation of both Earcons and Speech may be suitable substitutes for Existing Vehicle Sounds for information presentation without an adverse effect on driver workload. Automated scenarios produced a lower workload in comparison to manual scenarios $\left(\mathrm{F}_{1,13}=6.993, \mathrm{p}=0.02\right)$. Mean DALI results are shown in Table 1.

\begin{tabular}{|c|c|c|c|c|}
\hline & Existing & Earcons & Auditory Icons & Speech \\
\hline Man & 15.29 & 16 & 19 & 18 \\
\hline Auto & 10.71 & 14 & 22.57 & 20.79 \\
\hline
\end{tabular}

Table 1- Mean DALI workload scores for sound cue type during manual and automated driving conditions. Maximum possible score is 30 . (Lower $=$ better $)$

\section{SUS Analysis}

Usability was found to differ significantly between sound cue types $\left(\mathrm{F}_{3,39}=33.057, \mathrm{p}<0.001\right)$. Post hoc pair-wise comparisons showed Existing Vehicle Sounds produced higher usability scores compared to Auditory Icons $(\mathrm{p}<0.001)$ and Speech $(\mathrm{p}<0.001)$. Earcons also produced higher usability scores in comparison to Auditory Icons $(\mathrm{p}<0.001)$. Earcons were not found to score significantly higher usability scores compared to Speech. Comparisons between Auditory Icons and Speech were not found to be significantly different. These results reinforce our DALI findings and suggest that a decrease in usability results in an increase in workload when using Auditory Icons for primary driving task information in automated vehicles. The Speech sound cue type scored low for usability but did not cause a significant increase in workload. Both Existing Vehicle Sounds and Earcons did not differ significantly in usability. This implies that Earcons can offer a similar level of usability in automated vehicles for information presentation as Existing Vehicle Sounds. Manual driving scenarios did not produce significantly different usability scores than automated scenarios. Auditory Icons scored lowest during automated driving scenarios. During automated scenarios participants may have paid more attention to the sound cue types presented, as they were not burdened with any driving requirements. This may have impacted their responses to Auditory Icons resulting in negative emotional responses, lower satisfaction and higher distractions scores in comparison to other sound cue types. No significant interaction on usability between sound cue type and driving method was found. Table 2 shows that the mean usability score for Speech was higher during automated scenarios. This compliments both Existing Vehicle Sounds and Earcons that follow a similar trend. Auditory icons in contrast were found to receive a lower mean result during automated scenarios than manual.

\begin{tabular}{|c|c|c|c|c|}
\hline & Existing & Earcons & Auditory Icons & Speech \\
\hline Man & 80 & 64.46 & 33.75 & 39.46 \\
\hline Auto & 84.46 & 65.36 & 32.68 & 48.93 \\
\hline
\end{tabular}

Table 2 - Mean SUS scores for sound cue type. Maximum possible score is 100 . $($ Higher $=$ better $)$

\section{Likert-Scale Analysis}

Participants were presented with six 5-point Likert-scale rated questions between each scenario (see Procedure). Likert scales for questions 1 to 5 ranged from Strongly Disagree (1) to Strongly Agree (5). Question 6 ranged from Very Dissatisfied (1) to Very Satisfied (5). Manual and automated driving conditions were not found to differ significantly for any questions.

\section{Q1. Enhancing Awareness to Vehicle's Actions}

There was a significant difference between the 4 sound cue types $(\chi 2 \quad(5)=25.840, \mathrm{p}<0.001)$. Pairwise comparisons showed participant awareness to the vehicle's actions to be lower during scenarios containing Auditory Icons $(M=2)$ than Existing Vehicle Sounds $(\mathrm{Z}=-3.744, \mathrm{p}<0.001)(M=4)$ and Earcons $(\mathrm{Z}=-2.876, \mathrm{p}=0.004)(M=3.5)$. As Earcons scored similarly to Existing Vehicle Sounds, this highlights their effectiveness as a method of presenting primary driving information. Speech $(M=3)$ also provided a similar level of awareness to that of Existing Vehicle Sounds. 


\section{Q2. Enhancing Awareness to Own Actions}

A significant difference between the 4 sound cue types was found for enhancing awareness to participants own actions $(\chi 2(5)=21.491, \mathrm{p}=0.001)$. Pairwise comparisons showed that awareness was significantly higher during scenarios using Existing Vehicle Sounds $(M=4)$ in comparison with Auditory Icons $(\mathrm{Z}=-2.921, \mathrm{p}=0.003)(M=2)$. This result suggests that Earcons $(\mathrm{M}=3)$ and Speech $(\mathrm{M}=3)$ were satisfactory to ensure participants were aware of their own actions similar to Existing Vehicle Sounds. Only Auditory Icons were significantly less effective than all other sound cue types.

\section{Q3. Feeling of Control}

A difference between the 4 sound cue types was found ( $\chi 2$ $(5)=17.867, p=0.003)$. Participants felt less in control during scenarios containing Auditory Icons $(M=2)$ in comparison to Existing Vehicle Sounds $(Z=-1.747, \mathrm{p}<0.001)$ $(M=4)$ and Earcons $(Z=-2.707, \quad \mathrm{p}=0.007) \quad(M=3.5)$. Comparing Existing Vehicle Sounds, Earcons and Speech produced no significant differences suggesting that these sound cue types all produced a comparatively similar sense of control during both driving scenarios.

\section{Q4. Necessary Sound Cues}

A significant difference between the 4 sound cue types was found $\left(\chi^{2}(5)=45.631, \mathrm{p}<0.001\right)$. Participants felt the necessary sound cues were presented significantly better by the Existing Vehicle Sounds $(\mathrm{Z}=-4.155, \mathrm{p}<0.001)(M=5)$, Earcons $(Z=-3.991, \mathrm{p}<0.001)(M=4)$ and Speech $(Z=-3.136$, $\mathrm{p}=0.002)(M=4)$ in comparison to Auditory Icons $(M=2)$.

\section{Q5. Distraction to Primary Driving Task}

When asked whether the sound cue types in each scenario were a distraction to the primary driving task, a significant difference was found $(\chi 2(5)=47.183, \mathrm{p}<0.001)$. Pairwise comparisons showed that Auditory Icons were more distracting than Existing Vehicle Sounds $(\mathrm{Z}=-4.374$, $\mathrm{p}<0.001)(M=2)$ and Earcons $(\mathrm{Z}=-2.943, \mathrm{p}=0.003)(M=3)$. Speech $(M=2)$ was also more distracting than Existing Vehicle Sounds $(\mathrm{Z}=-3.829, \mathrm{p}<0.001)$. Earcons were also more distracting than Existing Vehicle Sounds $(\mathrm{Z}=-3.261$, $\mathrm{p}=0.001$ ).

\section{Q6. Sound Cue Type Satisfaction}

A significant difference between the 4 sound cue types was found in relation to participant satisfaction $(\chi 2(5)=60.551$, $\mathrm{p}<0.001)$. Participants were more satisfied with Existing Vehicle Sounds $(M=4)$ than Auditory Icons $(\mathrm{Z}=-4.425$, $\mathrm{p}<0.001)(M=2)$ and Speech $(\mathrm{Z}=-4.127, \mathrm{p}<0.001)(M=2)$. This suggests participants were most satisfied with sounds they were already most accustomed to from previous driving experience. Earcons $(M=4)$ were also more satisfying than both Auditory Icons $(\mathrm{Z}=-3.710, \mathrm{p}<0.001)$ and Speech $(\mathrm{Z}=-3.070, \mathrm{p}=0.002)$. Earcons were not significantly less satisfying than Existing Vehicle Sounds.

\section{Emotional Classification of Primary Driving Sounds}

We recorded primary emotions felt by participants towards the primary driving sounds presented after each scenario. Earcons and Existing Vehicle Sounds during both driving conditions elicited emotional responses such as "Satisfied", "Happy" and "Amused" corresponding to both passive and active positive quadrants in the emotion wheel. In contrast, the Auditory Icons and Speech most often elicited the opposite emotional responses, such as "Annoyed" and "Frustrated" situated in the active, negative quadrant. Only during the manual Speech scenario were modal emotional responses positive. A similar pattern was maintained for the other sounds braking, gear changing and indication although some outliers were also present. The modal outlier emotion for the gear changing sound during the manual Auditory Icons scenario was "Satisfied". The indicator sound also obtained an outlying modal emotional response in comparison to the other sounds. During the automated Speech scenario participants report feeling "Satisfied" which contrasts their reports for the other sounds: acceleration, braking/deceleration and gear-changing where the majority reported feeling "Annoyed".

\section{Subjective Responses}

Upon completion of Study 1 participants were asked to provide some questions in order to gauge their opinions of the different sound cue types. For example, participants were asked whether any sounds were absent during the study but had been expected. 10 out of the 14 participants highlighted they did not expect any other sounds to be heard. Out of the 4 that did, 2 highlighted they expected external vehicle sounds, with the remaining 2 stating they expected "smoother sounds" and "more differentiation between gear up \& gear down sounds". These responses highlight that participants were satisfied with the level of immersion provided by OpenDS and that the sound cue types presented the appropriate auditory cues similar to real-world driving situations.

\section{Summary Study 1}

In relation to our first objective for this study, we did not find any benefits over Existing Vehicle Sounds when using alternative sounds cue types. DALI workload and SUS usability scores were comparatively similar for Earcons. Our Likert scale analysis shows Earcons were also comparatively useful for enhancing awareness to the vehicle's actions as well as providing a sense of control. These results go some way to addressing our second and third objectives and suggest that while Existing Vehicle Sounds were most effective, it may also be possible to utilise Earcons in order to maintain a sense of control in automated vehicles. Furthermore, Earcons and Existing Vehicle Sounds did not differ in participant preference. As Earcons are synthesised tones, it is necessary to investigate further whether an improved approach to their design may in fact provide greater benefit than Existing Vehicle Sounds when conveying a sense of control in automated vehicles. 


\section{STUDY 2}

To explore the use of Earcons further, a second study was undertaken to address whether Earcons must be naturally mapped to their existing counterparts. Focusing this second study on Earcons was appropriate due to the complexity of dynamic sonic parameters implicated in the creation of Existing Vehicle Sounds. Since Earcons can be produced using singular sonic parameters, it would be possible to determine which of the parameters were most useful when designing Earcons and Existing Vehicle Sounds for automated vehicles. This study compared pitch, timbre, repetition and volume [4,5]. To achieve this, the methodology of study 1 was replicated with different Earcon types. The aim of study 2 was to answer the following 4 research questions:

S2.Q1: What sonic parameters are most appropriate when presenting artificial driving sounds in automated vehicles?

S2.Q2: Are alternative earcon mappings more effective than natural mappings when designing sounds to enhance driver awareness in an automated vehicle?

S2.Q3: Do earcons provide any benefits when used to present primary driving sounds in automated vehicles?

S2:Q4: Are mechanical driving sounds still necessary to ensure drivers are aware of the intended actions of an automated vehicle?

Study 1 implemented Earcons in a simplistic way following guidelines set out by Brewster et al. [7]. These Earcons were mapped to imitate existing vehicle sounds. For example, as the acceleration of the simulated vehicle varied, the pitch of the accelerator earcon fluctuated dynamically, in parallel to the RPM of the vehicle's engine. This natural mapping can be compared to alternative mapping methods that utilise findings from previous studies by Beattie et al. $[4,5]$ on drivers preference towards sonic parameters that catch their attention. New sets of Earcons were created based on these findings that altered by pitch, timbre, repetition, and volume and are discussed briefly in the following section.

\section{Sound Cue Types}

This study contained 2 primary sound cue types; Existing Vehicle Sounds and Earcons. However, different combinations of Earcons were created, these were manipulated by pitch, timbre, repetition and volume exclusively. Both the Existing Vehicle Sounds and Earcons from study 1 were re-used in study 2. 3 additional earcon sets were then created.

\section{Timbre Earcons}

This sound cue type featured sounds that varied in timbre. For instance, the acceleration and deceleration sounds varied as the accelerator pedal intensity varied. This was achieved by varying the intensity of two oscillating waveforms; one a simple sine wave oscillating at a frequency of $700 \mathrm{~Hz}$ and another saw-tooth wave oscillating at the same frequency. As the accelerator pedal was depressed the frequency of the waveforms increased in accordance with the engine RPM. Whilst at idle only the sine wave was heard whereas at full RPM only the saw tooth wave was heard. This gave the effect of the sound morphing from a mild, rounded sound to something considerably harsher sounding as the vehicle's RPM modulated. The gear changing sound featured two short synthesised saxophone tones. If the driver changed into a higher gear then the timbre of the second tone was processed to sound harsher. When changing to a lower gear the timbre of the first tone was processed to sound harsher. This was done to differentiate between the original pitch earcon and to provide information to the driver relating to the change in gear state. Finally the indicator sound varied over time as a synthesised sine-wave tone containing multiple harmonics with a fundamental frequency of $1500 \mathrm{~Hz}$. The longer the indicator was active then the more morphed the sound would become.

\section{Repetition Earcons}

Multiple audio files were used for the acceleration and deceleration repetition Earcons. Firstly, the earcon featured the sine wave tone with a frequency of $700 \mathrm{~Hz}$. At idle (700RPM) the tone repeated every quarter note at $120 \mathrm{bpm}$. In 1000 RPM intervals the frequency of the repetition was doubled. For instance at 1500RPM the tone repeated every eighth note at $120 \mathrm{bpm}$ and so on until max (7000RPM). The indicator sound was not altered during this sound cue type and operated in the same manner as during the Earcon sound cue type in study 1 . The two short synthesised saxophone tones used for gear changing sounds altered again depending on whether a higher or lower gear had been selected. If a higher gear had been selected the second tone from the sound was modulated to sound stuttered and repeated. When a lower gear had been selected the first tone featured a similar modulation.

\section{Volume Earcons}

The volume Earcons followed a similar method of execution to the pitch and timbre Earcons. At engine idle the volume of the acceleration and deceleration sounds were $-15 \mathrm{~dB}$ lower than the initial volume of the other Earcons in the study. As the engine RPM increased so too did the volume of the acceleration sound. At max RPM (7000RPM) the volume of the sound was $+3 \mathrm{~B}$ louder than the other Earcons at peak loudness. The indicator earcon increased in loudness the longer the duration it stayed activated for. The gear changing sounds followed a similar trend as used during the other earcon types. The synthesised saxophone tones altered in volume depending on whether a higher or lower gear was selected.

\section{Participants}

A new set of 14 participants ( 9 male, 5 female) aged 23 to 43 (mean=29) took part in the study, all with valid driving licenses. Their mean driving frequency range was 1 to 3 hours per week. 10 participants owned or had regular use of a vehicle. 3 participants were experienced with automatic transmissions and the rest with manual transmissions. Participants were recruited via email and word of mouth. 


\section{Procedure}

The same procedure as study 1 was followed. $4 \times 1$ minute training scenarios were undertaken prior to the main study so that participants could become accustomed to the sound cue types they would experience during the study. The study featured a 2x10 within subjects design meaning participants were required to undertake a total of 10 scenarios. Driving conditions were counterbalanced and a randomised order of sound cue types was presented to each participant to minimise any possible risk of order effects.

\section{Study 2 Results}

All data was analysed using the same as study 1. The exception being pair-wise comparisons for sound cue types were conducted with a Bonferroni correction applied, resulting in a significance level set at $\mathrm{p}=0.0071$.

\section{DALI Workload Analysis}

Sound parameter type produced a significant effect on workload $\left(\mathrm{F}_{4,52}=5.923, \mathrm{p}=0.001\right)$. Pairwise comparisons showed that Existing Primary Driving Sounds $(\mathrm{p}=0.012)$ and Timbre Earcons $(\mathrm{p}=0.014)$ produced significantly lower workload scores in comparison to Repetition Earcons. No other differences were found when comparing sound cue types. This shows that Pitch Earcons, Timbre Earcons and Volume Earcons did not cause a workload increase in comparison to Existing Vehicle Sounds. These findings suggest that the use of Repetition when designing earcons to present primary driving information hinders driver workload. The manipulation of Pitch, Timbre and Volume are acceptable without causing a negative impact on workload. Automated scenarios produced a lower workload in comparison to manual scenarios $\left(\mathrm{F}_{1,13}=\right.$ $36.606, \mathrm{p}<0.001)$. This result is expected as participants were required to physically interact with the driving simulator during manual scenarios. There were no interaction effects between sound parameter types and driving conditions when measuring driver workload. Mean DALI results are shown in Table 3.

\begin{tabular}{|l|l|l|l|c|c|}
\hline & Existing & Pitch & Timbre & Repetition & Volume \\
\hline Man & 15.29 & 16.78 & 16.29 & 21.1 & 18.43 \\
\hline Auto & 9 & 8.14 & 10.36 & 13.57 & 10.36 \\
\hline
\end{tabular}

Table 3 - Mean DALI workload scores for sonic parameters. Maximum possible value is 30 . (Lower $=$ better $)$

\section{SUS Analysis}

Usability was found to differ significantly between sound parameter types $\left(\mathrm{F}_{4,52}=9.931, \mathrm{p}<0.001\right)$. Post hoc pair-wise comparisons showed Existing Vehicle Sounds produced higher usability scores compared to Repetition Earcons $(\mathrm{p}=0.004)$ and Volume Earcons $(\mathrm{p}=0.005)$. Pitch Earcons and Timbre Earcons did not produce significantly lower scores than Existing Vehicle Sounds. This indicates that implementing natural mapping when designing earcons is beneficial in terms of usability. This also highlights that the use of these sonic parameters is effective without reducing usability as can be seen in the case of Volume and Repetition Earcons. This finding reinforces our DALI findings and suggests that Repetition used to manipulate earcons used for primary driving sounds is not effective. Pitch Earcons and Timbre Earcons proved to be as effective as Existing Vehicle Sounds in terms of usability. This shows that exploiting these particular parameters when designing earcons for primary driving sounds does not increase workload or effect usability. Manual driving scenarios did not produce significantly different usability scores than automated scenarios. Furthermore no significant interaction on usability between sound parameter type and driving method was found. Table 4 shows the mean usability score for each sonic parameter Earcon type.

\begin{tabular}{|l|l|l|l|c|c|}
\hline & Existing & Pitch & Timbre & Repetition & Volume \\
\hline Man & 78.93 & 64.11 & 57.86 & 32.68 & 42.14 \\
\hline Auto & 71.96 & 67.50 & 53.21 & 43.39 & 52.68 \\
\hline
\end{tabular}

Table 4 - Mean SUS scores for sonic parameters cue types. Maximum possible score is 100. $($ Higher $=$ better $)$

Likert-Scale Analysis

Participants were presented with the same six Likert scale questions as were used during Study 1.

\section{Q1. Enhancing Awareness to Vehicle's Actions}

There was a significant difference between the 5 sound scenarios $(\chi 2(6)=36.956, \mathrm{p}<0.001)$. Pairwise comparisons showed participant awareness to the vehicle's actions to be lower during scenarios containing Repetition Earcons $(M=2)$ than Existing Vehicle Sounds $(Z=-2.928, \mathrm{p}=0.003)$ $(M=4)$, Pitch Earcons $(Z=-3.964, p<0.001) \quad(M=4)$ and Timbre Earcons $(\mathrm{Z}=-2.985, \mathrm{p}=0.003)(M=4)$. These results mirror DALI workload and SUS results, suggesting that Repetition Earcons increase workload, as participants are less aware of the vehicle's intended actions. The other sonic parameters (Pitch, Timbre, and Volume) produced a similar level of awareness to that of Existing Vehicle Sounds.

\section{Q2. Enhancing Awareness to Own Actions}

Participants were significantly more aware of their actions during manual scenarios $(M=4)$ than automated scenarios $(M=3) \quad(\mathrm{Z}=-2.875, \quad \mathrm{p}=0.004)$. Participants "Somewhat Agreed" that Existing Vehicle Sounds $(M=4)$, "Neither Agreed nor Disagreed" that Pitch Earcons $(M=3)$, and "Somewhat Disagreed" that Timbre, Repetition and Volume Earcons $(M=2)$ enhanced awareness to their actions.

\section{Q3. Feeling of Control}

No sound scenarios were significantly different in improving participant's sense of control during both manual and automated scenarios.

\section{Q4. Necessary Sound Cues}

A significant difference between sound scenarios was found $(\chi 2(6)=37.738, p<0.001)$. Participants felt the necessary sound cues were better presented by Existing Vehicle Sounds $(\mathrm{Z}=-3.150, \mathrm{p}=0.002)(\mathrm{M}=4)$, Pitch Earcons $(\mathrm{Z}=-$ 4.185, $\mathrm{p}<0.001)(M=4)$ and Timbre Earcons $(\mathrm{Z}=-1.055$, $\mathrm{p}=0.002)(M=4)$ compared to Repetition Earcons $(M=2)$. This finding is in line with both DALI and SUS results and 
reinforces that the use of repetition in earcon modulation is not suitable. Pitch, Timbre and Volume $(M=3)$ were not significantly different to Existing Vehicle Sounds.

\section{Q5. Distraction to Primary Driving Task}

A significant difference was found between sound scenarios $(\chi 2(6)=31.460, \mathrm{p}<0.001)$. Pairwise comparisons showed Existing Vehicle Sounds were less distracting than Timbre Earcons $(\mathrm{Z}=-3.060, \mathrm{p}=0.002)(M=4)$, Repetition Earcons $(\mathrm{Z}=-3.302, \mathrm{p}=0.001)(M=4)$ and Volume Earcons $(\mathrm{Z}=-$ 3.113, $\mathrm{p}=0.002)(M=4)$. Repetition Earcons were also more distracting than Pitch Earcons $(Z=-3.167, p=0.002)(M=3)$. Pitch Earcons were not found to be more distracting that Existing Vehicle Sounds, suggesting these were least distracting during study 2.

\section{Q6. Sonic Parameter Satisfaction}

A significant difference between sound scenarios was found in relation to participant satisfaction $(\chi 2 \quad(6)=39.810$, $\mathrm{p}<0.001)$. Participants were more satisfied with Existing Vehicle Sounds $(M=4)$ than Timbre Earcons $(\mathrm{Z}=-3.315$, $\mathrm{p}=0.001)(M=2)$, Repetition Earcons $(Z=-3.584, \mathrm{p}<0.001)$ $(M=1)$ and Volume Earcons $(\mathrm{Z}=-3.450, \mathrm{p}=0.001)(M=2)$. Pitch Earcons $(M=3)$ were also more satisfying than Repetition Earcons $(Z=-2.738, \mathrm{p}=0.006)$. While most users stated they "Somewhat Disagree" that Pitch Earcons were satisfying, pairwise comparisons show they were not significantly less satisfying than Existing Vehicle Sounds.

\section{Emotional Classification of Primary Driving Sounds}

Existing Vehicle Sounds during both driving conditions elicited emotional responses such as "Satisfied", and "Relaxed" corresponding to the passive positive quadrant. Volume Earcons also follow a similar pattern eliciting a "Calm" response during both driving conditions. During manual scenarios Timbre Earcons and Pitch Earcons were both marked making participants feel "Satisfied". In contrast Repetition Earcons were marked as either "Frustrating" or "Annoying" in the active, negative quadrant. During both automated scenarios containing Timbre Earcons and Pitch Earcons participants also marked "Annoyed". A similar pattern was maintained for the other sounds braking, gear changing and indication. Gear changing was most often marked making participants feel "Annoyed" excluding Existing Vehicle Sounds where participants marked "Relaxed" during both manual and automated. During the automated Timbre Earcons scenario modal responses were "Amused". Finally, modal responses for indicator were "Annoyed" except in the case of Existing Vehicle Sounds and Repetition Earcons where participant responses were "Calm" and "Satisfied". This is interesting as it highlights that when repetition is used by earcons to convey a driving sound that is already repeating, participants responded positively.

\section{Summary Study 2}

Our results point towards both timbre and pitch being the most appropriate sonic parameters to use when present acceleration and deceleration Earcons. In the context of indication repetition was also found to be appropriate. To answer our second objective, naturally mapped Earcons are more effective than using unfamiliar abstract mappings such as repetition for acceleration sounds. In reference to objective 3, as our Earcons contained individual parameters only, none were significantly more beneficial than Existing Vehicle Sounds. It is feasible to suggest that currently, Existing Vehicle Sounds are still necessary to ensure drivers are aware of the intended actions of an automated vehicle. Conversely, by combining our results regarding individual sonic parameters for Earcons to produce a more complex set may produce benefits over Existing Vehicle Sounds. However, further analysis must first be conducted in order to ascertain whether this may be the case.

\section{DISCUSSION}

\section{Benefits to Artificial Driving Sounds (S1.Q1 \& S1.Q2)}

Our findings show that Earcons do not significantly increase driver workload in comparison to Existing Vehicle Sounds (see Table 1). Participants also reported Earcons as providing a similar level of usability to that of Existing Vehicle Sounds (see Table 2). Findings from the emotion wheel show that participants were most often, "Satisfied" and "Happy" and in one case "Amused" by the Earcons used to represent the primary driving sounds. This shows participants accepted Earcons as a useful sound cue type for presentation of primary driving task information during both manual and automated scenarios. In contrast, our findings highlight a number of drawbacks to the use of Auditory Icons and Speech in comparison to Existing Vehicle Sounds. Auditory Icons were reported as being a significant distraction to the primary driving task, which had a negative impact on workload (see Likert-Scale Q5 in Study 1 Procedure). Participants reported their emotional response to Auditory Icons as "Frustrated" and "Annoyed". Furthermore, Auditory Icons made participants feel significantly less in control during both driving conditions than any other sound cue type. Furthermore, due to the noncontinuous nature of Auditory Icons, their ability to convey primary driving task information may be limited hence the rise in workload and reduction in usability as seen in our results. Speech scored low for usability and was found to be significantly less usable than Existing Vehicle Sounds. However, workload was not increased when using Speech as a sound cue type (see Table 1). These findings contrast with those of McKeown [20] where the use of auditory icons and speech was favoured to convey information in vehicles.

\section{Driving Sound Preferences (S1.Q3, S2.Q3 \& S2.Q4)}

Participants found Existing Vehicle Sounds significantly more satisfying than Auditory Icons and Speech during both driving conditions. Existing Vehicle Sounds also scored lowest in terms of workload and highest for usability. These results indicate there may have been a preference towards the use of Existing Vehicle Sounds by participants in our study. However, Earcons scored comparatively the same suggesting they were equally preferred and as satisfying as 
Existing Vehicle Sounds. Earcons also did not cause a significant distraction during our first study unlike Auditory Icons and Speech. This further suggests that Earcons are a viable alternative to Existing Vehicle Sounds. Results from our second study suggest that when using earcons to present acceleration, braking/deceleration and gear changing sounds, the sonic parameters Pitch and Timbre are most appropriate to ensure workload is not increased significantly and sounds are usable. While Repetition Earcons scored lowest for both workload and usability, it may be preferable to present indication sounds modulated by this parameter to ensure driver's emotional responses are positive. Pitch Earcons were found to be comparable to Existing Vehicle Sounds in terms of satisfaction. They were also the least distracting of all sonic parameters and comparable to the distraction of Existing Vehicle Sounds. This suggests that modulating earcons using the sonic parameter pitch is useful when designing earcons to present primary driving information without causing distraction.

\section{Earcon Mapping (S2.Q1 \& S2.Q2)}

Modulating the Earcons by Pitch, Timbre and Volume produced significantly lower workload scores than Repetition Earcons (Table 3). This may have been due to Repetition as being an unfamiliar method of modulating Earcons in the context of acceleration, braking and gear changing. This evidence correlates to findings by Wogalter et al. [40] where participants prefer hearing either familiar Existing Vehicle Sounds or a hum mimicking Existing Vehicle Sounds. Not surprisingly, emotional responses for Repetition Earcons were most often negative except in the case of the indication sound where participants stated they were "Calm". This finding follows the trend of drivers expecting certain familiar driving sounds related to specific functions. Existing Vehicle Sounds, Pitch Earcons and Timbre Earcons also produced significantly higher usability scores than Repetition Earcons and Volume Earcons (Table 4). This finding highlights their usefulness as parameters to modulate when designing artificial driving sounds for automated vehicles. Moreover, as both Pitch Earcons and Timbre Earcons follow a natural mapping to Existing Vehicle Sounds, it is recommended that a natural mapping strategy be adhered to so sounds are usable and do not increase driver workload significantly.

\section{LIMITATIONS}

The development and creation of new human-machine interfaces for automated vehicles is a new and emerging field. Because of this, there are currently no best practices from which to conduct effective analysis. The use of an automated vehicle for real-world studies presents safety issues that are ameliorated through the use of a driving simulator such as OpenDS used in our studies. Our results show promise towards the use of Earcons as an alternative method of presenting primary driving auditory feedback in automated vehicles. The work presented serves as a framework and acts as a step in the direction towards effective situational awareness in automated vehicles.

\section{CONCLUSION}

This work investigated the use of artificial driving sounds for the presentation of primary driving task information in a driving simulator. Initial findings showed Existing Vehicle Sounds to be most satisfying and most effective at providing awareness to participants own actions during both driving conditions. Earcons scored comparatively well in terms of workload effect and usability than Existing Vehicle Sounds. This result indicates the utility of Earcons to present primary driving information in automated vehicles without any adverse effect on workload. In contrast, Auditory Icons were found to increase workload and cause significant distraction during both driving conditions. While Auditory Icons have been favoured to convey information in vehicles [20], our findings suggest they are not an effective solution when presentation of primary driving information is required. While Speech is used often in vehicles for presentation of secondary driving information, for instance GPS [8], our findings also suggest that using Speech for the presentation of primary driving information may not be a viable option due to the low usability scores found during study 1 .

Regarding the application of Earcons used as an alternative to Existing Vehicle Sounds, we recommend a natural mapping approach be applied so that driver workload is not increased and that sounds are usable. We also suggest that artificial acceleration, braking and gear changing sounds should be modulated primarily by Pitch and Timbre to ensure familiarity with Existing Vehicle Sounds is maintained. By following this recommendation, earcons used to present primary driving information in automated vehicles will ensure drivers are aware of the vehicles actions and will not be distracted by unfamiliar sounds.

Previous research has investigated simple tones, auditory icons and earcons as warning sounds in simulated manual vehicles $[17,26]$. To the best of our knowledge this is the first time a study comparing different sound cue types in simulated automated vehicles has been conducted. Our results indicate that when designing auditory displays in automated vehicles to enhance driver awareness to their actions, Existing Vehicle Sounds are currently the most appropriate. Earcons that are naturally mapped by incorporating the sonic parameter Pitch will provide similar benefits as Existing Vehicle Sounds for this purpose. These findings lend themselves to the development of current level 3 automated vehicles where the driver is required to be available for occasional control of the vehicle. Regarding the current lack of feedback for driver reassurance as highlighted by Kraus et al. [16], we have shown that an auditory display containing Earcons modulated by Pitch and Timbre is also an effective solution for maintaining awareness to the actions of an automated vehicle. This opens up new possibilities for auditory displays and highlights the use of Earcons as an effective alternative for information presentation in automated vehicles. 


\section{REFERENCES}

1. Adell, E., Varhelyi, A., \& Hjalmdahl, M. Auditory and Haptic Systems for In-Car Speed Management: A Comparative Real Life Study. Transportation Research Part F: Psychology and Behaviour, 11 (2008), 445 458.

2. Alvarez, I., Martin, A., \& Dunbar, J. Designing DriverCentric Natural Voice User Interfaces. Adj. Proceedings of AutomotiveUI, (2011), 42 - 49.

3. Baillie, L., Morton, L., Moffat, D., \& Uzor, S. Capturing the Response of Players to a Location-Based Game. Personal and Ubiquitous Computing, (2009), 13 -24 .

4. Beattie, D., Baillie, L., Halvey, M., \& McCall, R. Maintaining a Sense of Control in Autonomous Vehicles via Auditory Feedback. In Proceedings of Perceptual Quality of Systems Workshop, (2013).

5. Beattie, D., Baillie, L., Halvey, M., \& McCall, R. What's Around the Corner? Enhancing Driver Awareness in Autonomous Vehicles via In-Vehicle Spatial Auditory Displays. In Proceedings of NordiCHI'14., ACM Press (2014).

6. Brewster, S. A., Wright, P. C., \& Edwards, A.D.N. A Detailed Investigation into the Effectiveness of Earcons. First International Conference on Auditory Displays, Santa Fe, New Mexico, (1992).

7. Brewster, S. A., Wright, P. C., \& Edwards, A.D.N. An Evaluation of Earcons for Use in Auditory HumanComputer Interfaces. In Proceedings INTERCHI'93, (1993), $222-227$.

8. Brown, B., \& Laurier, E. The Normal, Natural Troubles of Driving with GPS. In proceeding of the SIGCHI Conference on Human Factors in Computing Systems, ACM Press (2012), 1621 - 1630.

9. Campbell, J. L., Richman, J. B., Carney, C., \& Lee, J.D. In-Vehicle Display Icons And Other Information Elements. Volume I: Guidelines. 2004.

10. Fagerlönn, J., Lindberg, S., \& Sirkka, A. Graded Auditory Warnings During In-Vehicle Use: Using Sound to Guide Drivers Without Additional Noise. Proceedings of the 4th International Conference on Automotive User Interfaces and Interactive Vehicular Applications (AutoUI '12), ACM Press (2012), 85-90.

11. Figueiredo, L., Jesus, I., Machado, J. T., Ferreira, J., \& de Carvalho, J.M. Towards the Development of Intelligent Transportation Systems. Intelligent Transportation Systems 88, (2001), 1206 - 2011.

12. Ibañez-Guzman, J., Laugier, C., Yoder, J. D., \& Thrun, S. Autonomous Driving: Context and State-of-the-Art. In Handbook of Intelligent Vehicles, Springer, London. (2012), $1271-1310$.
13. Inagaki, T., \& Itoh, M. Human's Overtrust in and Overreliance on Advanced Driver Assistance Systems: A Theoretical Framework. International Journal of Vehicular Technology 2013, (2013), 1-8.

14. Jamson, A. H., Merat, N., Carsten, O. M., \& Lai, F.C. Behavioural Changes in Drivers Experiencing HighlyAutomated Vehicle Control in Varying Traffic Conditions. Transportation Research Part C: Emerging Technologies 30, (2013), 116 - 125.

15. Janssen, C.P., Iqbal, S.T., and Ju, Y.-C. Sharing a Driver's Context With a Caller via Continuous Audio Cues to Increase Awareness About Driver State. Journal of experimental psychology. Applied, (2014).

16. Kraus, S., \& Althoff, M. Cognition and Emotion in Autonomous Cars. Intelligent Vehicles Symposium, IEEE (2009), 635 - 640.

17. Larsson, P., \& Opperud, A. Emotional and Behavioral Response To Auditory Icons and Earcons In DriverVehicle Interfaces'. In Proceedings of 21st Enhanced Safety of Vehicles Conference, (2009), 15 - 18.

18. Lin, C-T., Chiu, T-T., Huang, T-Y., Chao, C-F., Luang, W-C., Hsu, S-H., \& Ko, L.-W. Assessing Effectiveness of Various Auditory Warning Signals in Maintaining Drivers' Attention in Virtual Reality-based Driving Environments. Perceptual and motor skills 108, 3 (2009), 825-835.

19. Math, R., Mahr, A., Moniri, M. M., \& Müller, C. OpenDS: A New Open-Source Driving Simulator for Research. GMM-Fachbericht-AmE 2013., (2013).

20. McKeown, D. Candidates for Within-Vehicle Auditory Displays. Proceedings of ICAD, (2005), 182-189.

21. Moore, M.M., \& Lu, B. Autonomous Vehicles for Personal Transport: A Technology Assessment. Available at SSRN 1865047, (2011).

22. Mourant, R, R., \& Rockwell, T, H. Strategies of Visual Search by Novice and Experienced Drivers. Human Factors: The Journal of the Human Factors 14, (1972), $325-335$.

23. Mynatt, E, D. Designing With Auditory Icons. In Conference Fompanion on Human Factors in Computing systems, ACM Press (1994), 269-270.

24. NHTSA. Automated Vehicle Policy on Levels of Automation and Considerations for Research Progress. 2013. Available at: http://www.nhtsa.gov/About ${ }^{+}$ NHTSA/Press+Releases/U.S.+Department+of+Transpor tation+Releases+Policy+on+Automated + Vehicle + Devel opment.

25. Pauzie, A. A Method to Assess The Driver Mental Workload: The Driving Activity Load Index (DALI). Intelligent Transport Systems, IET, April (2008). 
26. Politis, I., Brewster, S. A., \& Pollick, F. Evaluating Multimodal Driver Displays Under Varying Situational Urgency. In Proceedings of 32nd annual ACM Conference on Human Factors in Computing Systems, ACM Press (2014), 4067 - 4076.

27. Pollard, J.K., Guthy, C., Hastings, a., Stearns, M.D., and Garay-Vega, L. Evaluation of Sounds for Hybrid and Electric Vehicles Operating at Low Speed. Proceedings of the Human Factors and Ergonomics Society Annual Meeting 56, 1 (2012), 2206-2210.

28. Sandberg, U., Goubert, L., and Mioduszewski, P. Are vehicles driven in electric mode so quiet that they need acoustic warning signals? August (2010).

29. Scott, J. J., \& Gray, R. A Comparison of Tactile, Visual, and Auditory Warnings for Rear-End Collision Prevention in Simulated Driving. Human Factors: The Journal of the Human Factors and Ergonomics Society 50, 2 (2008), 264-275.

30. Seagull, F. J., Xiao, Y., Mackenzie, C. F., \& Wickens, C.D. Auditory Alarms: From Alerting to Informing. Proceedings of Human Factors \& Ergonomics Society Annual Meetin 44, 1 (2000), pp. 223-226.

31. Shahab, Q., Terken, J., \& Eggen, B. Auditory Messages for Speed Advice in Advanced Driver Assistance Systems. In Proceedings of the 2nd International Conference on Automotive User Interfaces and Interactive Vehicular Applications, ACM Press (2010), $50-56$
32. Siciliano, B., Khatib, O., \& Groen, F. The DARPA Urban Challenge: Autonomous Vehicles in City Traffic. (2009).

33. Singh, S., Payne, S.R., and Jennings, P.A. Detection and emotional evaluation of an electric vehicle' $\mathrm{s}$ exterior sound in a simulated environment. (2013), 1-9.

34. Siwiak, D. and James, F. Designing Interior Audio Cues for Hybrid and Electric Vehicles. AES 36th International Conference, (2009).

35. Suzuki, K., \& Jansson, H. An Analysis of Driver's Steering Behaviour During Auditory or Haptic Warnings for the Designing of Lane Departure Warning System. JSAE Review, 24 (2003), 65 - 70.

36. Vilimek, R., \& Hempel, T. Effects of Speech and NonSpeech Sounds on Short-Term Memory and Possible Implications for In-Vehicle Use. In Proceedings of ICAD Workshop: "Combining Speech and Sound in the User Interface,” (2005).

37. Vollrath, M. Speech and Driving - Solution or Problem? IET Intelligent Transport Systems 1, 2 (2007), 89 - 94.

38. Walker, G, H., Stanton, N, A., \& Young, M, S. Where Is Computing Driving Cars? International Journal of Human-Computer Interaction 13, 2 (2001), 203-229.

39. Walker, G, H., Stanton, N, A., \& Young, M, S. The Ironies of Vehicle Feedback in Car Design. Ergonomics 49, 2 (2006), 161-79.

40. Wogalter, M.S., Lim, R.W., and Nyeste, P.G. On the hazard of quiet vehicles to pedestrians and drivers. Applied Ergonomics 45, 5 (2014), 1306-1312. 